\title{
SPR-spectroscopy of protein molecules adsorbed in microwave field
}

\author{
L.I.Berezhinsky, V.I.Chegel', Yu.M.Shirshov \\ Institute of Semiconductor Physics, NAS Ukraine, 45 Prospect Nauki, Kyiv, 03028, Ukraine \\ Phone: (38044) 26518 27; Fax: (38044) 26583 42; E-mail: che@isp.kiev.ua
}

\section{G.I.Dovbeshko}

Insitute of Physics, NAS of Ukraine, 46 Prospect Nauki, 03028 Kyiv, Ukraine

\section{O.V. Melnichuk}

Mykola Gogol State Pedagogic University, 2 Kropyv'yans'kogo St., Nizhyn, 16600, Ukraine

\begin{abstract}
A possibility to use surface plasmon resonance (SPR) in the investigations of the microwave radiation influence upon proteins in the process of their adsorption on the gold surface and their interaction with other proteins. For the first time, this method is applied to experimental study of physical parameters of the biological molecules under the action of the weak (from 1 to $10 \mathrm{~mW} / \mathrm{cm}^{2}$ ) electromagnetic fields in real-time regime. The changes in the process of adsorption of protein on the gold at different stages of the absorption as well as the changes in the reaction of antigen-antibody were introduced under the action of microwave field. More dense layers of protein was obtained in the microwave fields resulting in the decreased contribution of non-specific and specific interactions between proteins.
\end{abstract}

Keywords: surface plasmon resonance, adsorbed layer, protein, antigene-antibody, microwave field, polarization of medium, dielectric permeability, gold film.

Paper received 13.07.01; revised manuscript received 01.12.01; accepted for publication 12.12.01.

\section{Introduction}

It is well known that the surface excitation spectroscopy which deals with polaritons, plasmons, magnons, etc., is an extraordinarily sensitive method for studying the surface properties of crystals, films, molecules, and so on. In recent years, new interest to these methods appeared in connection with biological problems. The method of surface plasmon resonance (SPR) proved itself to be one of the most successful in studying interactions between the molecules [1]. Up to date, there are some important problems in biology, and among them the following: monitoring the protein interaction in immune reactions (of the "antigen - antibody" type), studying the interactions of "nucleic acid - nucleic acid" type when determining complementarity (or hybridization) of nucleic acid sequences, as well as the interaction between lipid and nucleic acid (for instance, when studying "virus - cell" interactions). These are not a full list of problems where SPR is actively used now. Various sensors using this method successfully operate nowadays [2]. Therefore, we turn to this method to firstly apply it when investigating the effect of the weak microwave (MW) radiation of millimeter range on biological molecules and reactions.
The problem of interaction of low intensity electromagnetic radiation of extremely high frequencies (EHF) or MW radiation with biological systems have not yet acquired a comprehensive attention from the side of academic sciences. However, due to its indisputable importance in applied medicine and biology, it is one of the most interesting for researchers who work in these fields [3-8]. Although any theory of interactions of MW radiation with biological objects is not currently developed through the deficiency of our knowledge concerning specifical mechanisms of transformations of energy of these waves in biomolecules, there are several noteworthy models partly concerning to such mechanisms [4-8]. Using the great experience in this field, the authors of the paper concentrated their efforts to experimentally reveal physical parameters of biological systems as well as physical-and-chemical properties of biomolecules that could be changed under action of weak electromagnetic fields in the mm-range.

\section{Materials and methods}

As objects for these investigations, we used proteins (albumin, immunoglobulin, anti-immunoglobulin) adsorbed 


\section{I. Berezhinsky et al.: SPR-spectroscopy of protein molecules adsorbed in ...}

on the surface of gold films and irradiated by low-intensity MW radiation. The SPR experiment was performed in accordance with the technique described in [2]. The MW radiation was introduced by teflon waveguide within the SPR cuvette from the top. We applied $1-10 \mathrm{mB} / \mathrm{cm}^{2}$ power density of $\mathrm{MW}$ radiation at the frequency of 60 GHz. As MW source was used G4-142 generator (Vilnius, USSR).

\section{Results and discussion}

The SPR method is based on the properties of surface waves in electron gas. Spreading along the interface of two media, these waves are in strong dependence on the dielectric permeability of the media [1], which can be easily seen from the following relation:

$k_{s p}=\frac{\omega}{c} \sqrt{\frac{\varepsilon(\omega) \varepsilon_{a}}{\varepsilon_{a}+\varepsilon(\omega)}}$.

where $k_{s p}$ is the wave vector of plasmon; $\varepsilon(\omega), \varepsilon_{a}$ are dielectric permeability of metal and dielectric thin films, respectively; $\omega$ and $c$ are common notations for frequency and velocity of light.

As it follows from Eq. (1), the wave vector of a surface plasmon excited at the metal-dielectric interface changes its value with dielectric permeability. If one places some biological molecules with a fixed $\varepsilon_{a}$ value onto the metal surface instead of dielectric, then one changes the conditions of spreading of surface plasmon.

When monitoring the angular position of the minimum in the surface plasmon spectrum under resonance conditions, one can monitor the kinetics of biological reaction, that is, obtain the so-called SPR-sensogram. If the conditions of plasmon resonance are changed as a result of the action of MW field on the adsorbed biological molecules, and the reaction antigen-antibody has another courses at different stages, then the role of MW radiation can be ascertained in these experiments. Earlier we showed that microwave field can be efficient in changing the dielectric permittivity of amino acid single crystals, as well as of the solutions of biological molecules and cells in visible and IR spectral ranges [9-11]. The interactions of molecules and cells can be changed in microwave fields, too [12].

At the beginning of this work, we repeated the experiment made by us earlier [13], where the MW field changed the adsorption of protein $\left(10^{-1}-10^{-3} \%\right.$ mioglobin solution in the phosphate buffer with $\mathrm{pH}=7.4$ ) on the metal surface (we used a silver in these experiments). In the experiment, the MW field $\left(50 \mathrm{~mW} / \mathrm{cm}^{2}\right.$, frequency $\left.37-53 \mathrm{GHz}\right)$ was turned on after saturation of the protein adsorption and for $10-15$ min resulted in shifting the minimum of SPR curve (SPR response) toward larger angles by several angular minutes. Turning the field on different stages of adsorption led to the similar shift of the SPR curve. These data enabled us to suggest that microwave field action resulted in densification of the adsorbed protein molecular layer.
We performed the same experiment with the use of more sensitive SPR device ("PLASMON SPR-4m" designed by the Institute of Semiconductor Physics of NAS of Ukraine). Its operation is based on excitation of a surface plasmon in a thin gold film. In the experiment, we observed 3 angular minute shift of the SPR response toward larger angles (Fig. 1), which was a result of the action of MW field (with power $5 \mathrm{~mW} / \mathrm{cm}^{2}$ ) for approximately 10 minutes after finishing the adsorption process. Here, as an object of investigations we used immunoglobulin $(0.3 \mathrm{~g} / 1$ protein solution in the phosphate buffer with $\mathrm{pH}=7.4$ ).

In another experiment, after achieving saturation of adsorption, we studied an influence of MW field on the course of adsorption of immunoglobulin from an identical solution for three hours; in this case, the shift toward large angles was ca. 200 arc seconds (i.e., 3.2 arc minutes) (Fig.2). Switching on the MW field at the point when adsorption has not yet reached its maximum value, but was close to it, resulted in the $4 . .5$ angular minute shift on the adsorption curve (see Fig. 3) and further run of the adsorption process.

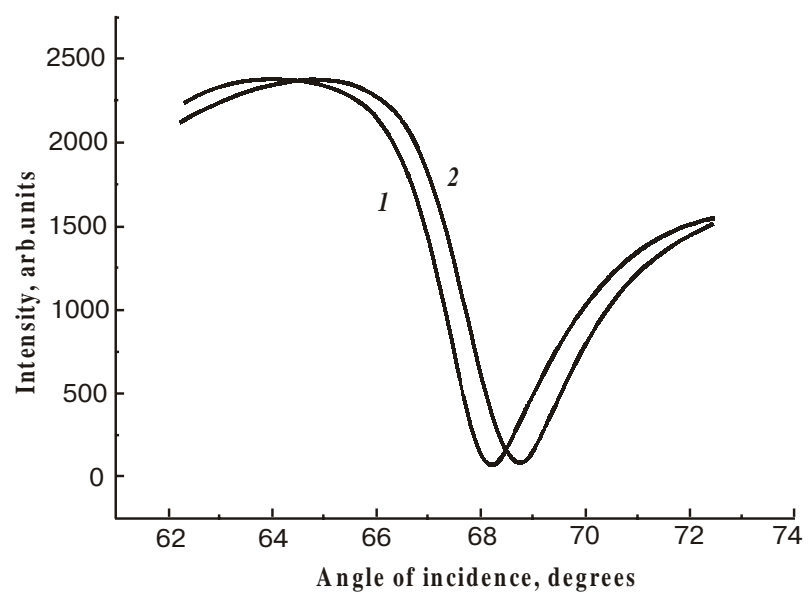

Fig. 1. A small power microwave field applied to a saturated layer of adsorbed protein shifts the SPR minimum toward large angles: 1 - before MW action; 2 - in the course of MW action.

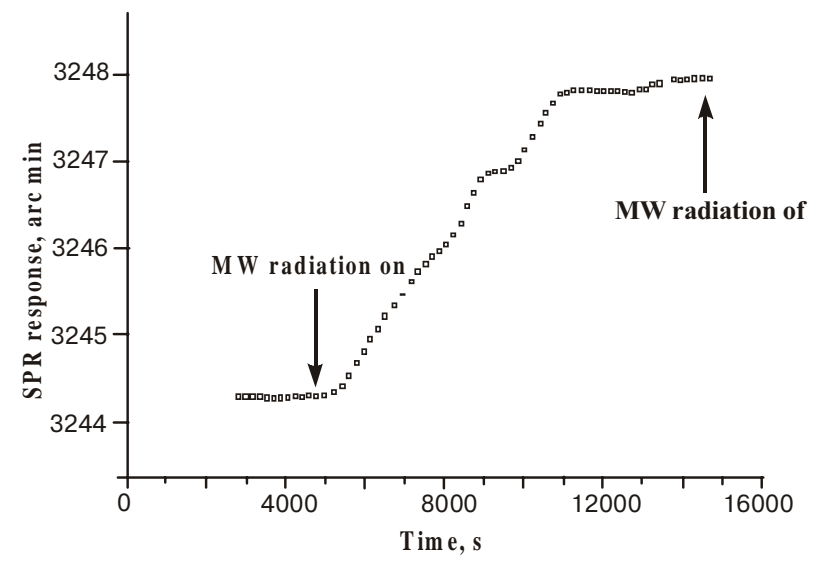

Fig. 2. Change of the position of SPR curve caused by applying a small power MW radiation to an adsorbed protein layer. 


\section{I. Berezhinsky et al.: SPR-spectroscopy of protein molecules adsorbed in ...}

Switching on the MW field at the other stages of the $\mathrm{IgG}-\mathrm{a}-\mathrm{IgG}$ reaction had no influence on the curve behavior (Fig 4). However, the resulting response showed a considerable contribution of non-specific interaction during this immune reaction. Described experiment was rather well reproducible.

As a result, an assumption emerged about decrease of contribution of specific interactions, which requires supplementary more careful investigation. In addition, there exist some unresolved questions: which processes are capable to shift the SPR curve? Could it be represented as a change of protein molecule packaging when applying the MW field?

The polarization of the medium, $P(t)$, under applying to it time-dependent electric field, $E(t)$, one can represent by the formula: $P(t)=\varepsilon_{0}\left(\varepsilon_{a}-1\right) E(t)$, where $\varepsilon_{0}$ and $\varepsilon_{a}$ are

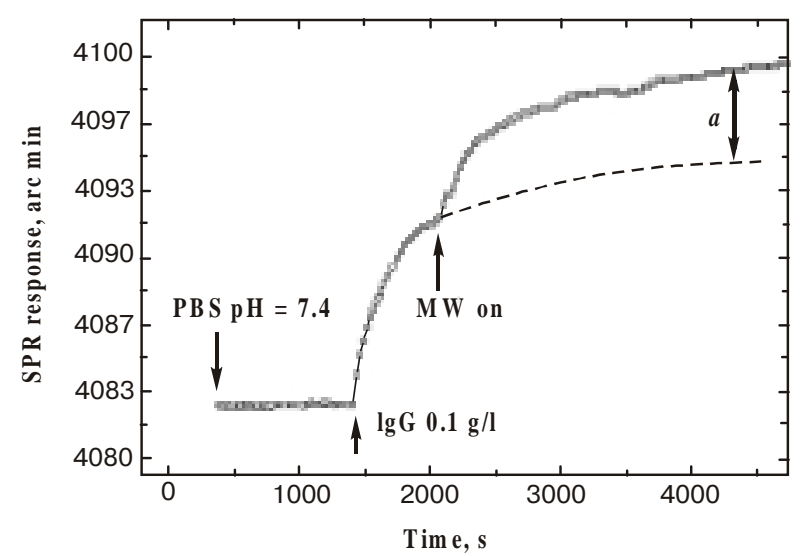

Fig. 3. Action of MW field during protein absorption at the stage before saturation results in a noticeable shift of the SPR minimum toward large angles.

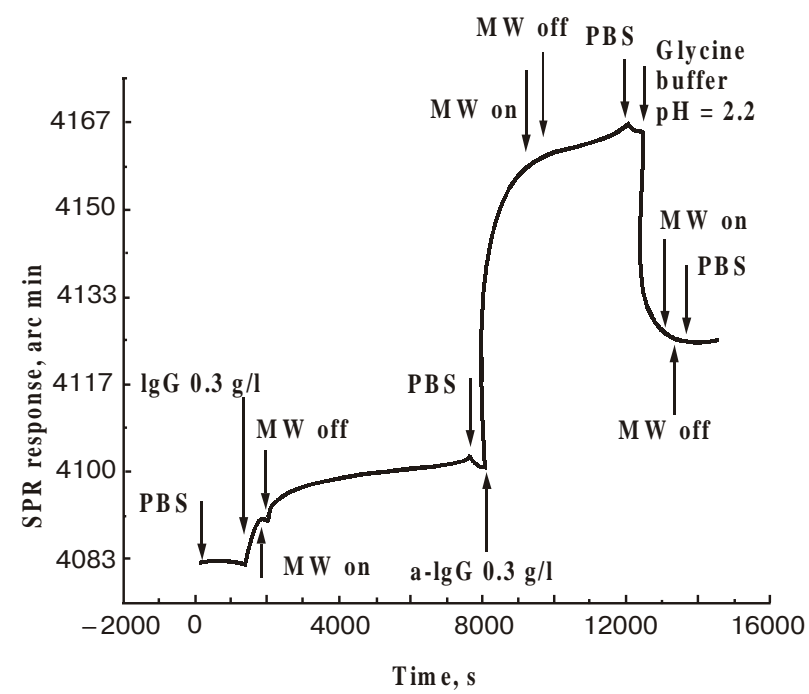

Fig. 4. SPR response under MW filled application at the different stages of immune reaction. respective dielectric permeability for vacuum and the sample, and $E(t)$ is an applied electric field. At the molecule level, two addends, $P_{m}$ and $P_{a}$, give contribution into $P(t)$. The former represents an orientational polarizability and corresponds for orientation of the dipole moments of unchanged molecules in the applied field against forces of thermal disordering. In this case the moving of charged particles can influence the solution electroneutrality resulting in the dipole moment contribution in the first addend.

The second term $P_{a}$, which is called induced polarization, characterizes the dipole moment $\mathrm{m}_{\mathrm{i}}$, ind induced by the field and induced polarizability $m_{i, \text { ind }}=d i E$. In general case, $P_{m}$ and $P_{a}$ are considered as linearly independent. $P_{a}$ follows the changes of $E(t)$ up to the far IR region in the contrast with $P_{m}$. Therefore, the polarization induced by this field $P_{a}=\varepsilon_{0}\left(\varepsilon_{\infty}-1\right) E$ is some constant value for a given material and it can be characterized as so-called dielectric permeability $\varepsilon_{\infty}$ at infinitely large frequency.

As a consequence, the time evolution of $P_{m}(t)=\varepsilon_{0}\left(\varepsilon_{a}-\right.$ $\left.-\varepsilon_{\infty}\right) E$ determines $P(t)$ and is a characteristic for the processes that take place when the MW field is applied. These processes can correspond to: molecule re-orientation, redistribution of the network of hydrogen bonds, charge transfer as well as kinetic relaxation processes [11-13]. When acting on water and protein molecules, the MW field orients them in the vicinity of the water-metal boundary, which changes the conditions for formation of protein layer near this boundary. It is certainly possible to imagine more dense package of the molecules in the protein layer, because of our introducing a supplementary periodic force that can act against the heat factor. Certainly, in these experiments, we should estimate the influence of ordinary heating on the SPR spectra. Irradiation of a cuvette containing protein with more powerful MW-field $\left(10 \mathrm{~mW} / \mathrm{cm}^{2}\right)$ led to heating the solution by approximately 1 to $5^{\circ} \mathrm{C}$. Irradiation of the buffer solution for 10-15 minutes in the MW field led to the shift of the SPR curve by 20-25 angular seconds toward smaller angles and then reached a stationary value (Fig. 5). As it

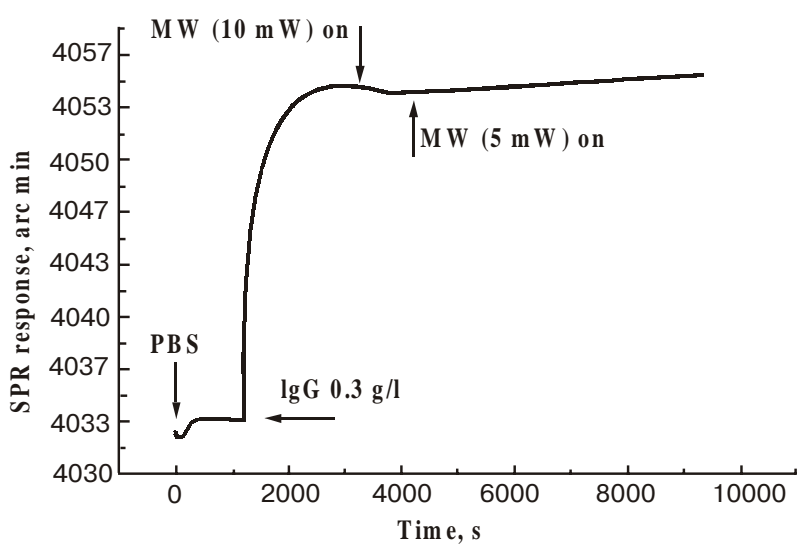

Fig. 5. The MW power density increasing causes to the shift of SPR minimum towards lower angles as a result of a heat of protein layer. 


\section{I. Berezhinsky et al.: SPR-spectroscopy of protein molecules adsorbed in ...}

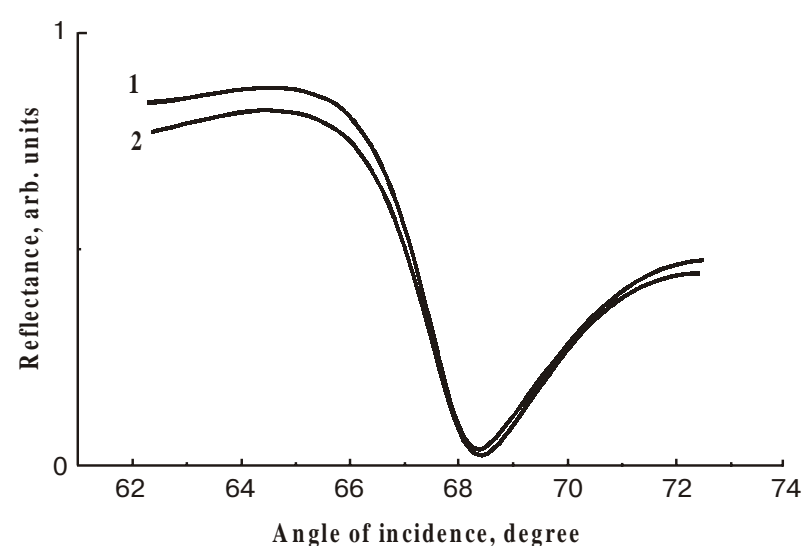

Fig. 6. Shift of the SPR curve toward smaller angles for heating of the molecular layer with MW radiation: 1 - before MW action 2 - after the long-term MW action

can be seen from the behavior of SPR sensogram for a buffer solution and protein, in the course of adsorption accompanied with MW field, they have opposite characters, although we cannot exclude some domination of the heating process above the ordering one in the MW field. Indeed, more durable heating process (for 10 hours or more, when irradiating with the most powerful field and observing protein adsorption) could lead to the shift of the SPR curve by 5 angular seconds into the side of smaller angles (Fig. 6).

Estimation showed that in the MW field the shift of SPR curve 2 by 6 arc minutes ( 0.1 degree) toward larger angles as compared with curve 1 (Fig 1). It corresponds to the change of the dielectric permeability characteristic of the protein molecule layer of $10 \mathrm{~nm}$ thickness by the value of 0.0433 (from 2.0592 up to 2.1025). For the case, the effective refraction coefficient before this $\mathrm{MW}$ field exposure was equal to 1.438 , and after it to 1.450 .

\section{Conclusions}

Therefore, the surface plasmon resonance effect enabled us to register the changes that take place in the protein layer during its adsorption on the gold surface at the presence of weak MW field. This field can stimulate recovering the adsorption process on both final adsorption stage and after its completion. On the basis of the SPR curve behavior and calculation, we made an assumption of protein layer densification when this layer is adsorbed at the presence of the MW field. In addition, we can conclude that the antigen-antibody reaction accompanied by switching on the MW field at its different stages can result in decreased contribution of both nonspecific and specific interactions in proteins due to more dense packing of the protein molecules.

\section{References}

1. S. Lofas et al. Bioanalysis with surface plasmon resonance. Sens.\& Act. B, B5, p. 113-121 (1991).

2. B.A. Snopok, K.V. Kostyukevych, O.V. Rengevych, Yu.M. Shirshov, E.F. Venger, I.N. Kolesnikova, E.V. Lugovskoi A biosensor approach to probe the stucture and function of the adsorbed protein: fibrinogen at the gold surface // Semiconductor Physics, Quantum Electronics \& Optoelectronics. 1, p. 121-134 (1998).

3. Ismailov E.S. Biophysical action of extra-high frequency radiation, Moscow, Energoizdat, 1987, p. 144 (in Russian)

4. Fundamental and applied aspects of application of millimeter electromagnetic radiation in biology and medicine, Proceedings of the First International Symposium with International Participation, - Kiev: Temporary Scientific Center, Cabinet of Ministers of UkrSSR, 1989, p. 404.

5. Lisitsa M.P. Physics of Alive 4, p.40-55 (1996).

6. Garibov P.E., Ostrovskiy A.V. // Uspekhi sovremennoi biologii 110(2), p.306-315 (1990) (in Russian).

7. Iskin V.D., Biological effects of millimeter waves and correlation methods of their detection. - Kharkov, Osnova, Kharkov University, p. 244 (in Russian) (1990).

8. Petrosyan V.I., Zhitneva E.A., Gulyaev Yu. V., Devyatkov N.D., Elkin V.A., Sinitsyn N.I.// Radiotekhnika 9, p.628-636 (1996)(in Russian).

9. Dovbeshko G., Berezhinsky L., Obuchovsky V. // Pгос. SPIE. 2795, p. 306-311 (1996)

10. Berezhinsky L.I., Dovbeshko G.I., Litvinov G.S., Obukhovsky V.V., Yanovskaya N.B., // Optics and Spectroscopy. 75(3), p.628-636 (in Russian) (1993).

11. Berezhinsky L.I., Gridina N.Ya., Dovbeshko G.I., Lisitsa M.P., Litvinov G.S., // Biophysics. 38, p.378-384 (1993) (in Russian).

12. Dovbeshko G., Berezhinsky L.. In: Book of Proceedings of 4th European Symposium on Electromagnetic Compatibility 2, p. 41 - 46, Sept. 11-15, Brugge, (2000),

13. Dovbeshko G.I, Berezhinsky L.I., Shirshov Yu.M.. In: Metal ions in Biological Systems, EUROBIC II, Florence, Italy, Aug. 30-Sept. 3, 1994, p. 49. 